\title{
Evaluation of the Relationship Between the Internal Acoustic Canal Anatomy and Loops of the Anterior Inferior Cerebellar Artery Using 3-Tesla Magnetic Resonance Imaging
}

\author{
Murat Bayav (iD ${ }^{1, *}$ and Murat Sahin ${ }^{1}$ \\ ${ }^{1}$ Department of Radiology, Eskisehir City Hospital, Eskisehir, Turkey \\ "Corresponding author: Department of Radiology, Eskisehir City Hospital, Eskișehir, Turkey. Tel: +90-5356171747, Email: muratbayav@hotmail.com
}

Received 2020 December 21; Revised 2021 September 17; Accepted 2021 September 19.

\begin{abstract}
Background: Anatomical variations in vascular structures are frequently encountered. In the cerebellopontine region, anatomical variations, especially variations in the anterior inferior cerebellar artery (AICA) in relation to cranial nerves passing through the internal acoustic canal (IAC), are common. Magnetic resonance imaging (MRI) provides detailed information for the evaluation of the cerebellopontine region and the IAC anatomy.

Objectives: This study aimed to examine the relationship between the IAC anatomy and AICA loop variations and to investigate the frequency of anatomical variations and its correlation with demographic variables.

Patients and Methods: In this study, 253 patients (age > 18 years), who underwent 3-Tesla MRI of the temporal bone between July 2019 and December 2019, were retrospectively evaluated. The exclusion criteria were images of poor diagnostic quality due to technical or patient-related reasons and the presence of a localized tumor that could disrupt the IAC. Four patients were excluded from the study because they had tumors related to IAC. The AICA variations were categorized according to the Chavda classification. The IAC size, canal diameter, canal length, and meatus width were measured, and the canals were characterized as cylindrical, funnelshaped, and bud-shaped.

Results: A total of 249 patients were included in this study. The Chavda type I vascular loop was the most common type on both sides. The mean size measurements of IACs were significantly higher in the group of vascular loops compared to the group without vascular loops. The presence of a vascular loop was significantly more common in funnel- and bud-shaped IACs compared to cylindrical IAC. Also, there was a negative correlation between the patient's age and all IAC dimensions.

Conclusion: The results of the present study showed that the AICA loop variations were closely related to the size and shape of the IAC.
\end{abstract}

Keywords: Vascular Loop, Anterior Inferior Cerebellar Artery, Internal Acoustic Canal, Temporal Bone, MRI

\section{Background}

Anatomical variations in vascular structures are frequently encountered. In the cerebellopontine region, anatomical variations, especially variations in the anterior inferior cerebellar artery (AICA) in relation to cranial nerves passing through the internal acoustic canal (IAC), are common (1). Magnetic resonance imaging (MRI) provides detailed information for the evaluation of the cerebellopontine region and the IAC anatomy (2). With recent advances in MRI technologies, small voxel sizes, acquired at a high magnetic field strength, have allowed for the optimal evaluation of anatomical features.

It is important to investigate and understand anatomical variations, especially before surgical procedures. T2- weighted (T2W) MRI with thin sections can successfully demonstrate the relationship between cranial nerves and vascular structures in patients with signs of neurovascular compression $(2,3)$. Previous studies have mostly examined the clinical symptoms of vascular loops of the AICA, but did not report any significant relationship (4-6). For example, Marques et al. evaluated the IAC morphology using computed tomography(CT) scan (7). However, there is no study in the literature on the relationship between the anatomical features of the IAC and AICA vascular loops.

\section{Objectives}

This study aimed to investigate the relationship between the IAC size and shape and the AICA variations in pa- 
tients without pathological IAC lesions and to determine the distribution of anatomical variations and measurements by age and gender. It can be helpful for head and neck surgeons to understand the IAC anatomical features and relations with AICA loops before surgeries, such as IAC bone dissection for tumor removal. Such knowledge can be also useful for radiologists and anatomists to describe the anatomical features properly.

\section{Patients and Methods}

\subsection{Patients}

In the present study, the images of patients, who underwent contrast-enhanced temporal bone imaging using a 3-tesla MRI scanner between July 1, 2019 and December 31, 2019, were evaluated retrospectively. Patients older than 18 years were included in this study. The exclusion criteria were images of poor diagnostic quality due to technical or patient-related reasons (e.g., motion artifacts and metalinduced artifacts) and the presence of a tumor that could disrupt the IAC.

The contrast-enhanced temporal bone MRI images of 253 patients who met the criteria were acquired in this study. Four patients were excluded from the study, because they had a tumor associated with the cerebellopontine corner or IAC; the data of 249 patients were finally evaluated (Figure 1). Before the onset of the study, the local ethics committee approval and institutional permission were obtained. Informed consent was not required by the ethics committee due to the retrospective design of the study.

\subsection{MRI Examination}

Images were acquired using a Siemens Magnetom Skyra $^{\text {TM }}$ 3T MRI device (Siemens AG, Munich, Germany). After injecting $15 \mathrm{cc}$ of an intravenous contrast agent (gadobutrol), fat-saturated $\mathrm{T1W}$ images were obtained in the axial plane according to the temporal bone imaging protocols with the following parameters: repetition time (TR), $8.4 \mathrm{~ms}$; echo time (TE), $3.69 \mathrm{~ms}$; number of excitations (NEX), 1; section thickness, $1 \mathrm{~mm}$; matrix size, $288 \times 264$; and field of view, $180 \times 180 \mathrm{~mm}$. Also, to acquire T2W sampling perfection with application-optimized contrasts using different flip angle evolution (SPACE) images, the parameters were as follows: TR, $1200 \mathrm{~ms}$; TE, $154 \mathrm{~ms}$; NEX, 2; flip angle, $120^{\circ}$; echo train length, 69 ; section thickness, 0,6 $\mathrm{mm}$; matrix size, $320 \times 300$; and field of view, $160 \times 160$ $\mathrm{mm}$.

\subsection{Imaging Analysis}

The images were evaluated by two radiologists (with six and two years of experience, respectively) based on consensus. The IAC was evaluated in terms of the presence of vascular loops using contrast-enhanced axial images on both sides. The contrast-enhanced T1W images provided a better contrast of the AICA to background and cranial nerves compared to T2W images (Figure 2). Patients with vascular loops were categorized according to the Chavda classification (3). According to this classification, a type 1 loop borders the internal auditory meatus, but does not enter the canal; a type 2 loop extends to the medial half of the canal; and a type 3 loop extends to the lateral half of the canal (Figure 3). The evaluation of the canal shape and dimensions was based on axial T2W SPACE images. The canals were also classified as cylindrical, funnel-shaped, and budshaped (Figure 4). The IAC measurements were performed in millimeters $(\mathrm{mm})$ at $3 \times$ magnification (Figure 5).

\subsection{Statistical Analysis}

After the demographic data, collected from the patients' hospital records, and the imaging findings were registered on a computer, statistical analysis was performed in IBM SPSS Version 20.0 (IBM Corp., Armonk, NY, USA). Continuous data with a normal distribution are expressed as mean \pm standard deviation (minimum/maximum) and categorical data as frequency and percentage. A correlation analysis was performed using Pearson's correlation test for parametric data to determine the correlation of the IAC diameter with the patient's age. The mean IAC diameters were compared between different groups (gender, age group, canal shape, and Chavda classification) using independent samples t-test. Besides, categorical data, such as canal shape and Chavda classification, were compared using chi-square test. $\mathrm{P}<0.05$ was considered statistically significant.

\section{Results}

Of 249 patients included in the study, 145 (58.2\%) were female, and 104 (41.8\%) were male. The mean age of the patients was $45.65 \pm 13.07$ years (range: 18 - 80 years); the mean age of women was $43.84 \pm 12.73$ years (range: 18 - 73 years); and the mean age of men was $48.16 \pm 13.18$ years (range: 18 - 80 years).

On the right side, no vascular loop was observed in 153 (61.4\%) patients. However, type 1 vascular loops were found in 60 (24.1\%) cases, type 2 loops in 24 (9.6\%) cases, and type 3 loops in 12 (4.8\%) cases. On the left side, no vascular loop was observed in 146 (58.6\%) cases, while type 1 vascular loops were found in 65 (26.1\%) cases, type 2 in 24 (9.6\%) 

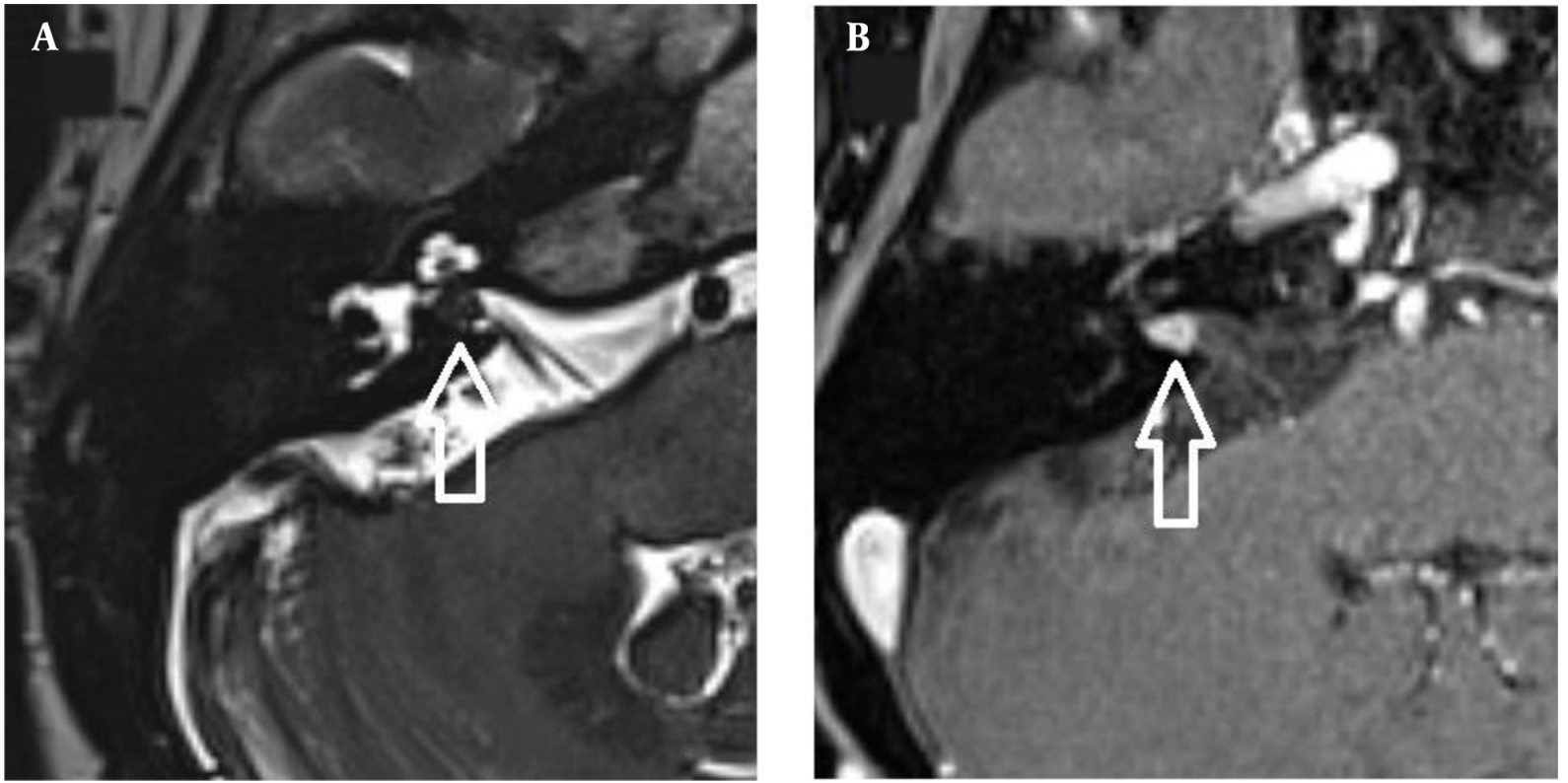

Figure 1. A, Axial T2W; and B, Axial contrast-enhanced T1W images of a 71-year-old female patient, indicating a schwannoma (arrows) located in the internal acoustic canal (IAC).
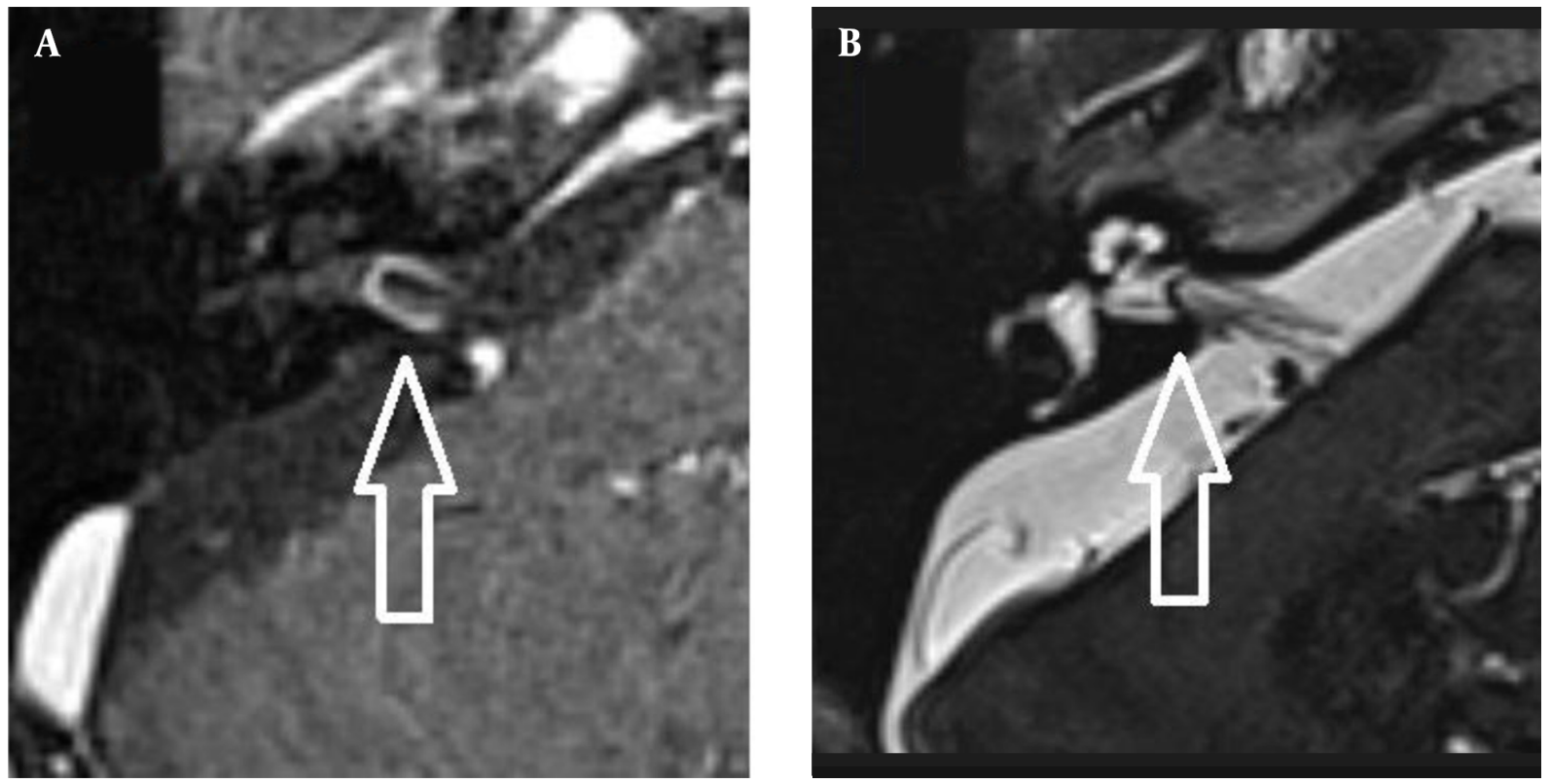

Figure 2. A, An anterior inferior cerebellar artery (AICA) loop on a contrast-enhanced T1W image; and B, The same loop on a T2W image (arrow).

cases, and type 3 in 14 (5.6\%) cases. There was no significant difference between the right and left ears in terms of the presence of a vascular loop $(\mathrm{P}=0.915)$.

In 498 inner ears, the mean diameter of the IAC was $4.56 \pm 1.03 \mathrm{~mm}$ (range: $1.9-8.59 \mathrm{~mm}$ ); the mean canal length was $9.62 \pm 1.59 \mathrm{~mm}$ (range: 5.25 - $15.56 \mathrm{~mm}$ ); and the mean meatus diameter was $6.02 \pm 1.86 \mathrm{~mm}$ (range: $2.82-13.54 \mathrm{~mm}$ ). In men, the mean diameter of the IAC was $4.46 \pm 1.03 \mathrm{~mm}$, the mean canal length was $9.41 \pm 1.62$ $\mathrm{mm}$, and the mean meatus diameter was $5.83 \pm 1.84 \mathrm{~mm}$, 

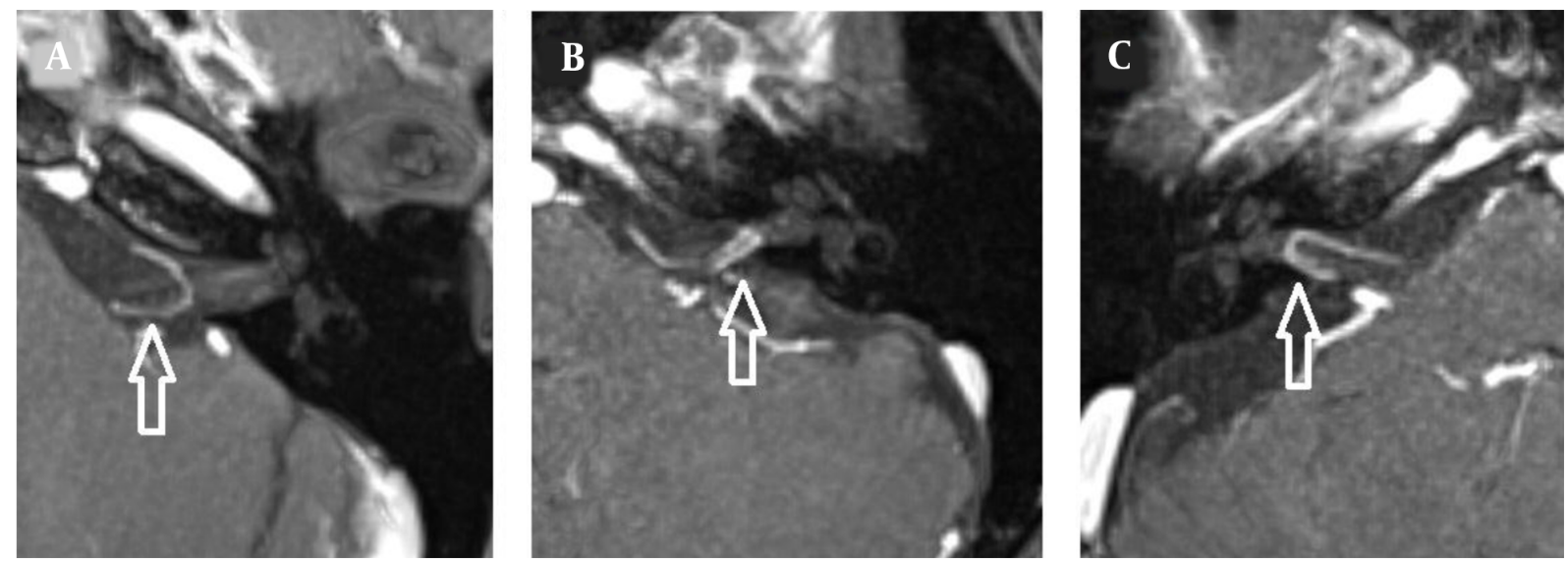

Figure 3. Maximum intensity projection for axial contrast-enhanced T1W images with a slice thickness of 2 mm, indicating an anterior inferior cerebellar artery (AICA) loop (arrows). A, a Chavda type 1 loop; B, a Chavda type 2 loop; and C, a Chavda type 3 loop.
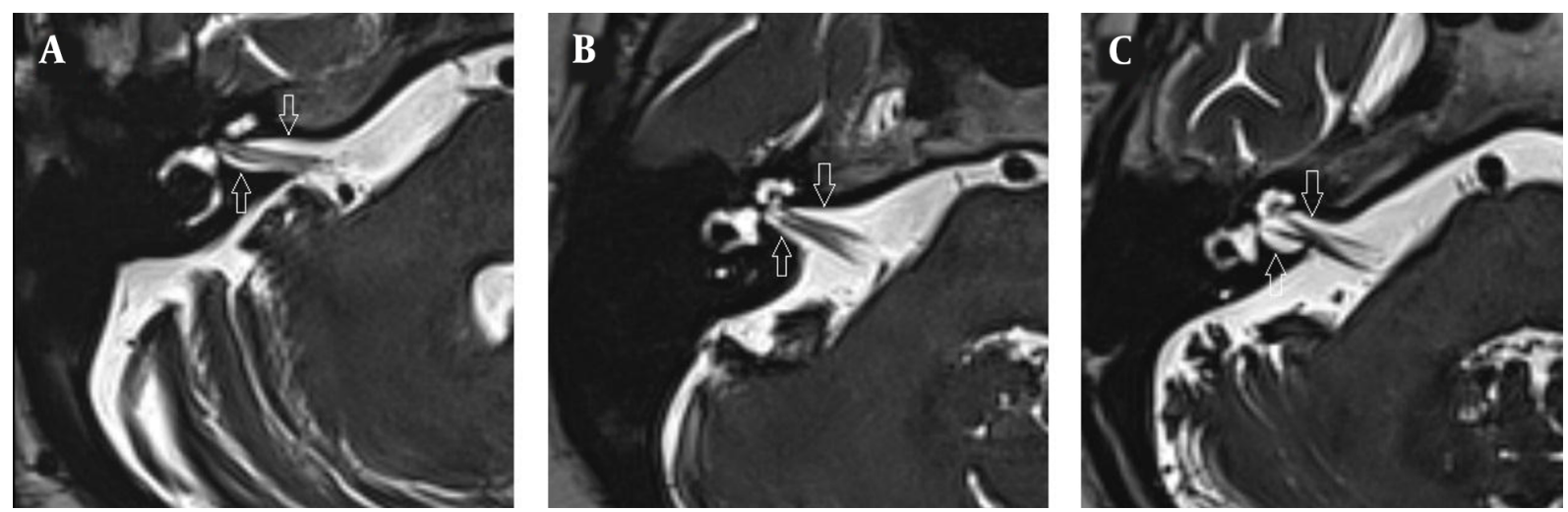

Figure 4. T2W images indicating the internal acoustic canal (IAC) shape (anterior and posterior borders are shown with arrows). A, Cylindrical IAC; B, Funnel-shaped IAC; and C, Bud-shaped IAC.

while in women, the mean corresponding values were 4.64 $\pm 1.00 \mathrm{~mm}, 9.77 \pm 1.54 \mathrm{~mm}$, and $6.15 \pm 1.86 \mathrm{~mm}$, respectively. All diameters of the inner ear were higher in women compared to men; however, only the mean IAC diameter was significantly higher $(\mathrm{P}=0.011$ for the IAC diameter, $\mathrm{P}=$ 0.059 for the meatus diameter, and $\mathrm{P}=0.058$ for the canal length).

On the right side, the mean IAC diameter was $4.50 \pm$ $0.99 \mathrm{~mm}$, the mean canal length was $9.39 \pm 1.62 \mathrm{~mm}$, and the mean meatus diameter was $5.82 \pm 1.84 \mathrm{~mm}$, while on the left side, the mean IAC diameter was $4.63 \pm 1.03 \mathrm{~mm}$, the mean canal length was $9.86 \pm 1.52 \mathrm{~mm}$, and the mean meatus diameter was $6.22 \pm 1.86 \mathrm{~mm}$. The canal length and meatus diameter were slightly higher on the left side compared to the right side $(\mathrm{P}=0.001$ and $\mathrm{P}=0.018$, respectively). Of all IACs, 230 (46.2\%) were cylindrical, 169 (33.9\%) were funnel-shaped, and 99 (19.9\%) were bud-shaped. The funnel-shaped and bud-shaped IACs were associated with the presence of a vascular loop $\left(\chi^{2}=12.688, \mathrm{P}=0.048\right)$ (Table 1).

In cases without vascular loops, the mean diameter of the IAC was $4.25 \pm 0.096 \mathrm{~mm}$, the mean canal length was $9.38 \pm 1.59 \mathrm{~mm}$, and the mean meatus diameter was 5.60 $\pm 1.73 \mathrm{~mm}$, while the mean corresponding values in the group of vascular loops were $5.03 \pm 0.91 \mathrm{~mm}, 9.99 \pm 1.51$ $\mathrm{mm}$, and $6.65 \pm 1.87 \mathrm{~mm}$, respectively. All of the IAC measurements were significantly higher in the group of vascular loops ( $\mathrm{P}<0.0001$ for all) (Table 2$)$. The IAC diameter and canal length showed weak negative correlations with the patient's age, while the meatus diameter had a moderate negative correlation with age $(\mathrm{r}=-0.479, \mathrm{P}<0.0001$; $\mathrm{r}=-0.387, \mathrm{P}<0.0001$; and $\mathrm{r}=-0,598, \mathrm{P}<0.0001$, respectively). Finally, when the patients were divided into two age groups of $\geq 65$ and $<65$ years, no significant difference 


\begin{tabular}{|c|c|c|c|c|c|}
\hline Canal shape & No loops & Chavda type 1 & Chavda type 2 & Chavda type 3 & Total \\
\hline Cylindrical & $152(30.5)$ & $54(10.8)$ & $15(3)$ & $9(1.8)$ & $230(46.2)$ \\
\hline Funnel-shaped & 99 (19.9) & $42(8.4)$ & $20(4)$ & $8(1.6)$ & $169(33.9)$ \\
\hline Bud-shaped & $48(9.6)$ & $29(5.8)$ & $13(2.6)$ & $9(1.8)$ & $99(19.9)$ \\
\hline Total & $299(60)$ & $125(25.1)$ & $48(9.6)$ & $26(5.2)$ & $498(100)$ \\
\hline
\end{tabular}

${ }^{\mathrm{a}}$ Values are expressed as No. $(\%)\left(\chi^{2}=12.688, \mathrm{P}=0.048\right)$.

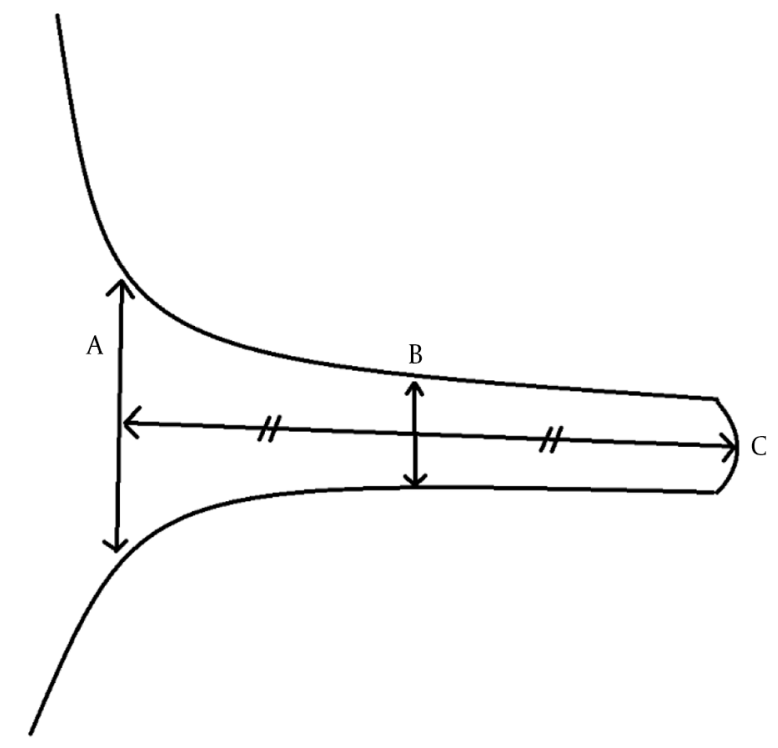

Figure 5. Schematic representation of the internal acoustic canal (IAC). A, Meatus width; B, Canal diameter; and C, Canal length.

was found between them in terms of the presence of a vascular loop $\left(\chi^{2}=1.214, \mathrm{P}=0.271\right)$.

\section{Discussion}

In the present study, in line with the literature, the most common type of IAC-associated AICA loop was Chavda type 1, in which the arterial loop is located outside the IAC on both sides. Type 1 vascular loops were followed by type 2 and type 3 loops $(4,6,8,9)$. According to the present results, all IAC measurements showed a negative correlation with the patient's age. Similarly, in a CT study, Marques et al. (7) found that the dimensions of the IAC were significantly larger in the pediatric group compared to the adults. However, we found no significant difference between the elderly patients aged $\geq 65$ years and others in terms of the presence of AICA vascular loops; this suggests that tortuosity and ectasia, which may occur in vas- cular structures with age, are not related to the presence of an AICA vascular loop.

In this study, the most common IAC shape was cylindrical (46.2\%), followed by funnel (33.9\%) and bud (19.9\%) shapes. In this regard, Marques et al. measured the frequency of funnel-shaped, cylindrical, and bud-shaped IAC and found differences in the IAC shape compared to the literature, which could be attributed to racial differences and anatomical structures affected by genetic changes during embryogenesis (7). The current study is the first to investigate the IAC shape in a Turkish population. The mean diameter of the IAC was $4.56 \pm 1.03 \mathrm{~mm}$ (range: $1.9-8.59 \mathrm{~mm}$ ), the mean canal length was $9.62 \pm 1.59 \mathrm{~mm}$ (range: 5.25 $15.56 \mathrm{~mm}$ ), and the mean meatus diameter was $6.02 \pm 1.86$ mm (range: 2.82 - $13.54 \mathrm{~mm}$ ).

In previous studies, the IAC dimensions have been examined radiologically by CT scan. Marques et al. found that the mean anteroposterior diameter of the IAC was $4.82 \mathrm{~mm}$, the mean canal length was $11.17 \mathrm{~mm}$, and the mean meatus diameter was $7.53 \mathrm{~mm}$ (7). Differences in the IAC size may be related to genetic and racial differences (similar to the shape of the canal). Based on the comparison of the IAC shapes regarding the presence of AICA vascular loops, the rate of vascular loops was higher in the funnel-shaped and bud-shaped IACs compared to cylindrical canals. This finding suggests that an arterial vascular loop may be related to the canal shape; however, there is no similar study in the literature.

In the present study, we focused on the anatomical features of the AICA and IAC rather than clinical symptoms related to AICA loop variations. So far, no significant relationship has been reported between arterial vascular loops and clinical symptoms, such as tinnitus or vertigo in previous studies (4-6). On the other hand, evaluating the IAC anatomy and variations before a surgical procedure, especially for tumors, not only facilitates tumor resection, but also preserves the labyrinth (10). Therefore, the IAC and AICA variations should be evaluated although they cause no clinical symptoms.

In previous studies, 3D T2W and constructive interference in steady-state (CISS) images were acquired to evalu- 


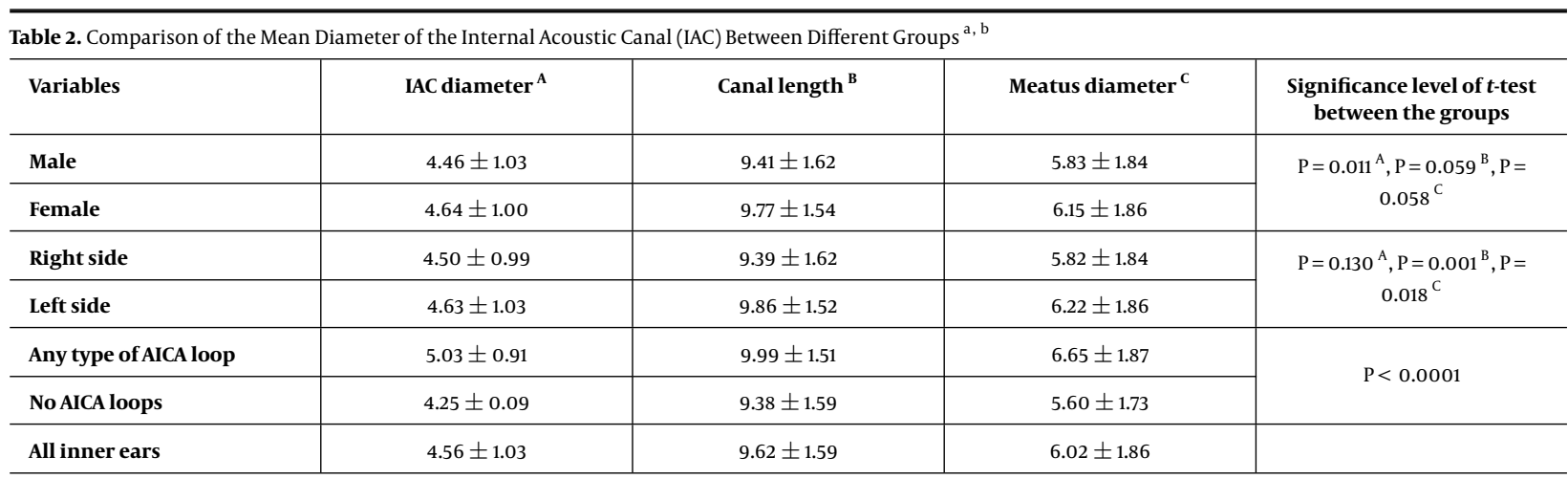

Abbreviations: IAC, internal acoustic canal; AICA, anterior inferior cerebellar artery.

${ }^{a}$ Values are expressed as mean $\pm \mathrm{SD}(\mathrm{mm})$.

${ }^{\mathrm{b}}$ P values for each variables are shown with capital letters (A-C).

ate the AICA anatomy $(9,11)$. Leal et al. prospectively studied trigeminal nerve vascular compression with a combination of 3D T2W, contrast-enhanced T1W, and time of flight (TOF) sequences and reported vascular compression with high inter-observer reliability. On contrast-enhanced T1W images, the arteries showed good visualization due to higher signal intensity (12). Also, in the present study, the AICA was more visible in contrast-enhanced images compared to T2W images.

Contrast-enhanced T1W imaging is recognized as the standard protocol, along with 3D T2W and TOF sequences, especially for neurovascular compression detection (13). Accordingly, the present study aimed to improve the visualization of vascular structures using intravenous contrast-enhanced images in temporal bone MRI examinations and to detect tumors that can cause anatomical impairments. On the other hand, the borders of the IAC are better visualized on T2W images, which is a disadvantage of T1W imaging. Therefore, the presence of vascular loops was only evaluated on T1W images in this study, while the canal shape and dimensions were evaluated on T2W images. Besides, a slice thickness of $0.6 \mathrm{~mm}$, provided by the 3T MRI machine, facilitated a better anatomical evaluation by $\mathrm{T} 2 \mathrm{~W}$ images as compared to previous studies using slice thicknesses of 0.7 to $0.8 \mathrm{~mm}(9,11,14)$.

The limitations of this study include its single-center design that limited the generalizability of our findings. Besides, due to the retrospective design of the study, the clinical symptoms could not be evaluated, and no imaging follow-up could be performed. Finally, the IAC measurements and shape evaluations were performed by two radiologists based on consensus; therefore, interobserver agreement could not be investigated.

In conclusion, knowledge of anatomical variations is important in surgical procedures. In previous studies, the AICA variations were evaluated clinically and symptomati- cally, while their relations with the anatomical features of IAC were not compared. The current study revealed a relationship between the size and shape of the IAC and the AICA loop variations. The present results showed that the AICA loop variations were closely related to the IAC shape and diameter.

\section{Footnotes}

Authors' Contribution: The authors contributed equally to the manuscript.

Conflict of Interests: The authors declare no conflict of interest.

Data Reproducibility: The data that support the findings of this study are available from the corresponding author.

Ethical Approval: This study was carried out according to the ethical standards and principles of the Declaration of Helsinki and approved by the Ethics Committee of Eskisehir Osmangazi University, Eskisehir, Turkey (approval code: 2020/291).

Funding/Support: The authors did not receive funding from any profit or non-profit organizations for the research, authorship, or publication of this article.

Informed Consent: The ethics committee waived the requirement for informed consent due to the retrospective design of the study.

\section{References}

1. Ouaknine GE. The arterial loops of the pontocerebellar angle. Microsurgical anatomy and pathological consideration. Adv Otorhinolaryngol.1982;28:121-38. doi:10.1159/000406750. [PubMed: 6981294].

2. Casselman JW, Kuhweide R, Deimling M, Ampe W, Dehaene I, Meeus L. Constructive interference in steady state-3DFT MR imaging of the inner ear and cerebellopontine angle. AJNR Am J Neuroradiol. 1993;14(1):47-57. [PubMed: 8427111]. [PubMed Central: PMC8334438]. 
3. McDermott AL, Dutt SN, Irving RM, Pahor AL, Chavda SV. Anterior inferior cerebellar artery syndrome: Fact or fiction. Clin Otolaryngol Allied Sci. 2003;28(2):75-80. doi: 10.1046/j.1365-2273.2003.00662.x. [PubMed: 12680822].

4. Gultekin S, Celik H, Akpek S, Oner Y, Gumus T, Tokgoz N. Vascular loops at the cerebellopontine angle: Is there a correlation with tinnitus? AJNR Am J Neuroradiol. 2008;29(9):1746-9. doi: 10.3174/ajnr.A1212. [PubMed: 18653684]. [PubMed Central: PMC8118805].

5. Hoekstra CE, Prijs VF, van Zanten GA. Diagnostic yield of a routine magnetic resonance imaging in tinnitus and clinical relevance of the anterior inferior cerebellar artery loops. Otol Neurotol. 2015;36(2):35965. doi: 10.1097/MAO.0000000000000279. [PubMed: 24691509].

6. Beyazal Celiker F, Dursun E, Celiker M, Durakoglugil T, Beyazal M, Inecikli MF, et al. Evaluation of vascular variations at cerebellopontine angle by 3D T2WI magnetic-resonance imaging in patients with vertigo. J Vestib Res. 2017;27(2-3):147-53. doi: 10.3233/VES-170616. [PubMed: 29064830].

7. Marques SR, Ajzen S, D. Ippolito G, Alonso L, Isotani S, Lederman H. Morphometric analysis of the internal auditory canal by computed tomography imaging. Iran J Radiol. 2012;9(2):71-8. doi: 10.5812/iranjradiol.7849. [PubMed: 23329967]. [PubMed Central: PMC3522352].

8. Makins AE, Nikolopoulos TP, Ludman C, O'Donoghue GM. Is there a correlation between vascular loops and unilateral auditory symptoms? Laryngoscope. 1998;108(11 Pt 1):1739-42. doi: 10.1097/00005537199811000-00027. [PubMed: 9818836].

9. Erdogan N, Altay C, Akay E, Karakas L, Uluc E, Mete B, et al. MRI assessment of internal acoustic canal variations using 3D-FIESTA sequences. Eur Arch Otorhinolaryngol. 2013;270(2):469-75. doi: 10.1007/s00405-
012-1994-7. [PubMed: 22456810].

10. Cueva RA, Chole RA. Maximizing Exposure of the Internal Auditory Canal Via the Retrosigmoid Approach: An Anatomical, Radiological, and Surgical Study. Otol Neurotol. 2018;39(7):916-21. doi: 10.1097/MAO.0000000000001866. [PubMed: 29995012].

11. Kazawa N, Togashi K, Ito J. The anatomical classification of AICA/PICA branching and configurations in the cerebellopontine angle area on 3D-drive thin slice T2WI MRI. Clin Imaging. 2013;37(5):865-70. doi: 10.1016/j.clinimag.2011.11.021. [PubMed: 23751264].

12. Leal PR, Hermier M, Souza MA, Cristino-Filho G, Froment JC, Sindou M. Visualization of vascular compression of the trigeminal nerve with high-resolution 3T MRI: A prospective study comparing preoperative imaging analysis to surgical findings in 40 consecutive patients who underwent microvascular decompression for trigeminal neuralgia. Neurosurgery. 2011;69(1):15-26. doi: 10.1227/NEU.0b013e318212bafa. [PubMed: 21346659].

13. Haller S, Etienne L, Kovari E, Varoquaux AD, Urbach $\mathrm{H}$, Becker M. Imaging of Neurovascular Compression Syndromes: Trigeminal Neuralgia, Hemifacial Spasm, Vestibular Paroxysmia, and Glossopharyngeal Neuralgia. AJNR Am J Neuroradiol. 2016;37(8):1384-92. doi: 10.3174/ajnr.A4683. [PubMed: 26892985]. [PubMed Central: PMC7960264].

14. Sirikci A, Bayazit Y, Ozer E, Ozkur A, Adaletli I, Cuce MA, et al. Magnetic resonance imaging based classification of anatomic relationship between the cochleovestibular nerve and anterior inferior cerebellar artery in patients with non-specific neuro-otologic symptoms. Surg Radiol Anat. 2005;27(6):531-5. doi: 10.1007/s00276-005-0015-6. [PubMed: 16322942]. 